Editora Omnis Scientia

ANAIS DA I JORNADA NORTE-NORDESTE DE ENGENHARIAS (ONLINE)

Volume 1

$1^{\text {a }}$ Edição

TRIUNFO - PE 


\section{Coordenador Científico}

Alex Aguiar da Silva

\section{Coordenador de Publicação}

Daniel Luís Viana Cruz

\section{Coordenadora do Evento}

Andréa Telino Gomes

\section{Organizadores}

Academics - Eventos acadêmicos online

Editora Omnis Scientia

Andréa Telino Gomes

Daniel Luís Viana Cruz

Alex Aguiar da Silva

\section{Palestrantes}

Alex Aguiar da Silva

André Búrigo Leite

Cecília Aguiar da Silva

Eugênio Bastos da Costa

Jorge Recarte Henriquez Guerrero

Renan Ferreira da Rocha

\section{Avaliadores}

Alex Aguiar da Silva

Helio Cardoso Martim

Péricles Felipe Bastos Gomes

\section{Imagem de Capa}

Freepik

\section{Revisão}

Os autores

\section{(9) $\mathbb{\oplus \Theta \Theta}$}

Este trabalho está licenciado com uma Licença Creative Commons - Atribuição-NãoComercialSemDerivações 4.0 Internacional.

O conteúdo abordado nos artigos, seus dados em sua forma, correção e confiabilidade são de responsabilidade exclusiva dos autores. 


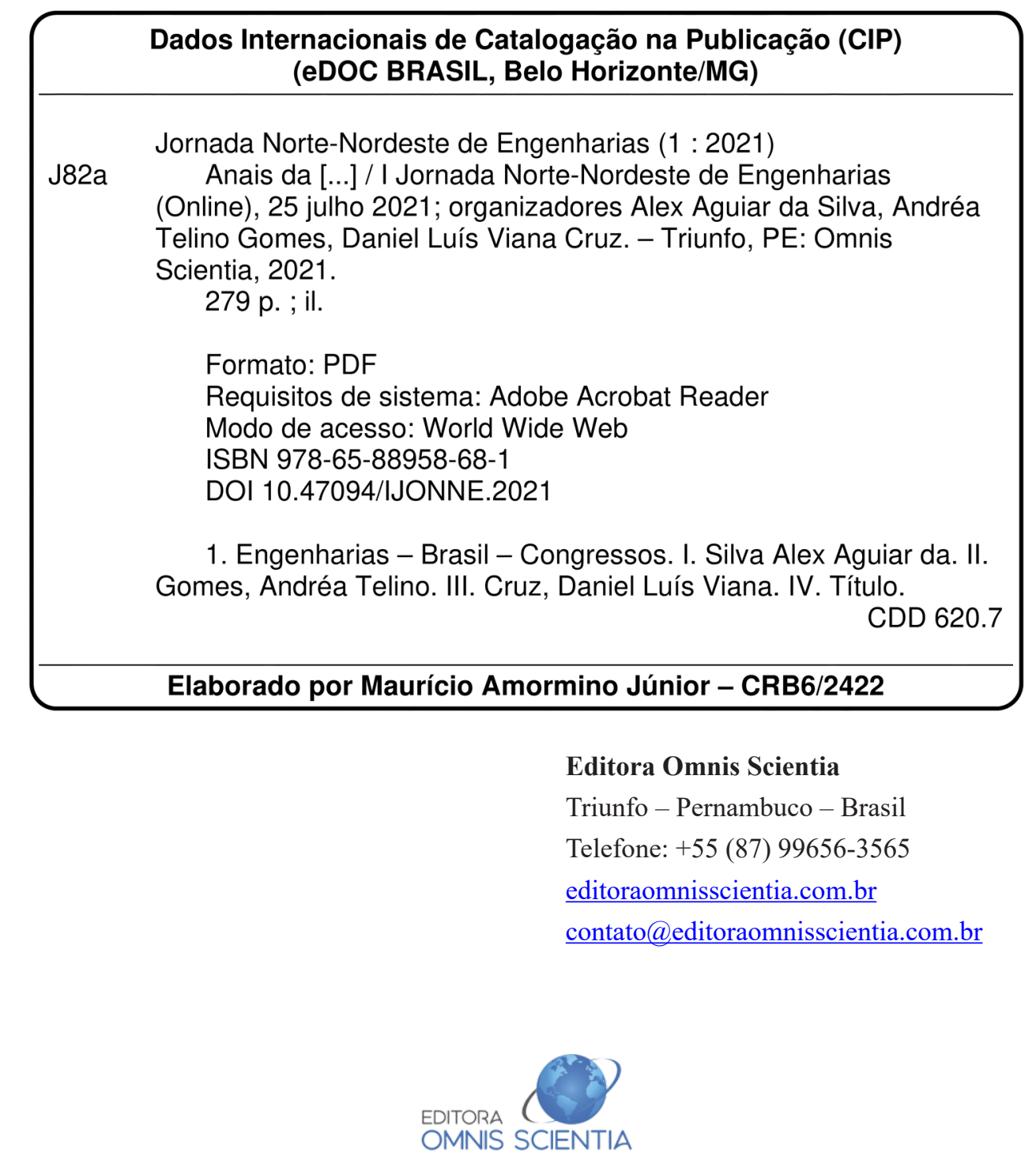




\section{EDITORIAL}

A grande área das engenharias abrangente uma série de ramos específicos, cada qual com determinados campos de aplicação e tipos de tecnologias, conjugando conhecimentos especializados no sentido de viabilizar as utilidades, tendo em conta a sociedade, a técnica, a economia e o meio ambiente. Deste modo, as engenharias aplicam o conhecimento científico, econômico, social e prático, para criar, desenhar, construir, manter e melhorar estruturas, máquinas, aparelhos, sistemas, materiais e processos. Assim, se adquire e se aplicam os conhecimentos matemáticos e técnicos na invenção, aperfeiçoamento e implementação de utilidades que realizem uma função ou objetivo.

A I Jornada Norte-Nordeste de Engenharias (online) I JONNE, objetivou reunir e integrar as engenharias para promover e apoiar esta área do conhecimento por meio de palestras, submissões de trabalhos na modalidade de resumos expandidos e exposição dos resumos aprovados.

Os participantes receberam certificados de participação de 20 horas e foi concedido menção honrosa para os três melhores trabalhos. Seguem os títulos dos resumos que foram premiados:

Modalidade resumo expandido

387210 - DIMENSIONAMENTO DE TROCADOR DE CALOR A PLACAS PARA PASTEURIZAÇÃO DE LEITE PELO PROCESSO HTST

387222 - DESENVOLVIMENTO DE UM DISPENSER DE ÁLCOOL GEL AUTOMÁTICO PARA MONITORAMENTO DA COMPLACÊNCIA DE MÃOS

387225 - REDE NEURAL ARTIFICIAL PARA VISCOSIDADE E CONDUTIVIDADE TÉRMICA DO FLUIDO REFRIGERANTE R32

A equipe organizadora da I JONNE agradece a todos os participantes, palestrantes e avaliadores pela participação e colaboração no congresso. 


\section{SUMÁRIO}

\section{ENGENHARIA CIVIL}

ANÁLISE DO USO DE RESÍDUO DE BRITAGEM DAS ROCHAS EM CONCRETO AUTOADENSÁVEL: UMA REVISÃO DA LITERATURA.....................................................12

ESTRATÉGIAS PARA A PREVENÇÃO DE ACIDENTES NA CONSTRUÇÃO CIVIL: UMA REVISÃO SISTEMÁTICA DA LITERATURA.

RESISTÊNCIA À COMPRESSÃO DE MISTURAS DE SOLO-CIMENTO COM NANOSSÍLICA E SÍLICA ATIVA.

PATOLOGIAS EM OBRAS PARALISADAS: O CASO DO ESTÁDIO COLOSSO DO TAPAJÓS NA CIDADE DE SANTARÉM - PA.

DIFERENÇA NO CUSTO DE MÃO DE OBRA EM FABRICAÇÃO DE LAJES PRÉ- MOLDADAS EM AMBITO NACIONAL

ESTUDO COMPARATIVO DE FUNDAÇÕES PARA UM EDIFÍCIO MODELO EM JOÃO PESSOA/PB: SAPATA X ESTACA HÉLICE CONTÍNUA.

MANIFESTAÇÕES PATOLÓGICAS EM EDIFICAÇÕES COM PAREDES DE CONCRETO ARMADO: ESTUDO DE CASO EM OBRA NA CIDADE DE JOÃO PESSOA - PB.....

REUTILIZAÇÃO DO PÓ RESIDUAL DE MARMORARIA PARA SUBSTITUIÇÃO DO AGREGADO MIÚDO NO CONCRETO.

SUBSIDÊNCIA OCORRIDA NOS BAIRROS PINHEIRO, BEBEDOURO E MUTANGE EM MACEIÓ - AL: UMA REVISÃO BIBLIOGRÁFICA.

CARACTERIZAÇÃO FÍSICA DAS VARIAÇÕES DIMENSIONAIS DO BAMBU GUADUA WEBERBAUERI DE RIO BRANCO - AC.

ESTUDO DA OCORRÊNCIA DE SOLOS COLAPSÍVEIS NA REGIÃO NORDESTE DO BRASIL.

OS EFEITOS DO USO DE POÇOS ARTESIANOS NO PROCESSO DE SALINIZAÇÃO COSTEIRA NA CIDADE DE SÃO LUÍS: UM LEVANTAMENTO BIBLIOGRAFICO.

TEORIADOS SÓLIDOS CELULARES EMPREGADOS EM REFORÇOS PARAACONSTRUÇÃO CIVIL

AVALIAÇÃO DO EXTRATO DE BOLDO E AROEIRA COMO INIBIDOR VEGETAL DE BAIXO CUSTO......

ANÁLISE DE DOSAGEM DE MATRIZ CIMENTÍCIA COM FIBRAS DE POLIPROPILENO: REVISÃO DA LITERATURA 
ANÁLISE DA RETENÇÃO DE CLORETOS EM ARGAMASSAS COM RESÍDUOS DE CELULOSE.

\section{ENGENHARIA DE MATERIAIS E METALÚRGICA}

ANÁLISE DA DEGRADAÇÃO FERRÍTICA DO AÇO INOXIDÁVEL DUPLEX SAF 2205 SUBMETIDO A ENVELHECIMENTO ISOTÉRMICO.

ANÁLISE DE INCLUSÕES E QUANTIFICAÇÃO DE POROSIDADE DO BRONZE AO ESTANHO EM ESTRUTURA BRUTA DE FUSÃO. 100

SÍNTESE RÁPIDA DE NANOFIOS DE NIOBATO DE SÓDIO. 105

AVALIAÇÃODAINCORPORAÇÃODACAREPADELAMINAÇÃOCOMOPARTEDAMATÉRIAPRIMA NA PRODUÇÃO DE SÍNTER DE FINOS DO MINÉRIO DE FERRO. 110

ESTUDO PROSPECTIVO DO PETRÓLEO APLICADO EM NANOMATERIAL CERÂMICO...... 115

ESTUDO PROSPECTIVO DE NANOMATERIAL CERÂMICO APLICADO EM SAÚDE MÉDICA E DENTÁRIA.

ESTUDO PROSPECTIVO DE MAGNETITA NANOMÉTRICA COMO MATERIAL FERROMAGNETICO.

ESTUDO PROSPECTIVO DE CERÂMICA AVANÇADA UTILIZANDO DOPAGEM......

BUSCA DE ANTERIORIDADE DE CERÂMICA APLICADA NA REMOÇÃO DE CONTAMINANTE..... .131

APLICAÇÕES DE $\mathrm{MoO}_{3}$ OBTIVO ATRAVÉS DE PRECIPITAÇÃO. 136

REVISÃO BIBLIOGRÁFICA SOBRE TUNGSTATO DE BÁRIO EM MEIO AQUOSO. 140

ESTUDOS DA DIFUSÃO DE CARBONO NOS AÇOS SAE 1020 E 1045 SUBMETIDOS À TRATAMENTO TERMOQUÍMICO DE CEMENTAÇÃO SÓLIDA. 145

\section{ENGENHARIA DO PRODUTO}

DESENVOLVIMENTO DE UM DISPENSER DE ÁLCOOL GEL AUTOMÁTICO PARA MONITORAMENTO DA COMPLACÊNCIA DE MÃOS. 150

\section{ENGENHARIA ELÉTRICA}

ENERGIA EÓLICA NO CEARÁ .156

\section{ENGENHARIA HIDRÁULICA}

ESTUDO DE MÉTODOS ANALÍTICOS PARA DETERMINAÇÃO DE PERDA DE CARGA EM TUBULAÇÕES DE PVC UTILIZADAS EM IRRIGAÇÕES. 162 
DESENVOLVIMENTO DE PRÁTICAS LABORATORIAIS APLICANDO OS CONCEITOS DE FENÔMENOS DE TRANSPORTES. 168

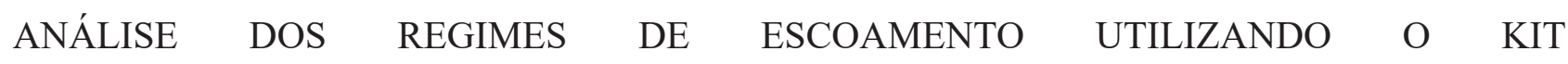
AQUALIBRIUM...

\section{ENGENHARIA MECÂNICA}

AVANÇO NO EMPREGO DE GABARITOS DE SOLDAGEM NO PROCESSO DE FABRICAÇÃO DE CHASSIS FORMULA SAE..

ANÁLISE DA DISTRIBUIÇÃO DE TEMPERATURA NOS PNEUS DE UM VEÍCULO FORMULA SAE PARA DIFERENTES CARGAS VERTICAIS. 184

\section{ENGENHARIA QUÍMICA}

UTILIZAÇÃO DE OXIDAÇÃO AVANÇADA PARA O TRATAMENTO DE EFLUENTES INDUSTRIAIS: UMA REVISÃO DA LITERATURA..

ANÁLISE DOS PRINCIPAIS PROCESSOS DE PRODUÇÃO DE BIODIESEL: UMA REVISÃO DE LITERATURA.

A QUÍMICA DOS AGROTÓXICOS USADOS EM AGRICULTURA: UMA REVISÃO DA LITERATURA. 201

AVALIAÇÃO CINÉTICA DA REAÇÃO DE DEGRADAÇÃO DE DIURON PRESENTE EM ÁGUAS SUPERFICIAIS.

ESTUDO DE CASO DO TRATAMENTO DE GÁS ÁCIDO COM COLUNA DE ABSORÇÃO DE LEITO RECHEADO.

ELABORAÇÃO DA CURVA DE CALIBRAÇÃO PARA ACRILAMIDA PELO MÉTODO DE CROMATOGRAFIA LÍQUIDA DE ALTA EFICIÊNCIA.

DETERMINAÇÃODECONSTANTESCINÉTICASPELOS MÉTODOSINTEGRALEDERUNGEKUTTA PARA REAÇÃO DE OXIDAÇÃO DO ANTRACENO.

DIMENSIONAMENTO DE TROCADOR DE CALOR A PLACAS PARA PASTEURIZAÇÃO DE LEITE PELO PROCESSO HTST.. 229

REDE NEURAL ARTIFICIAL PARA VISCOSIDADE E CONDUTIVIDADE TÉRMICA DO FLUIDO REFRIGERANTE R32.

TRANSFORMAÇÃO DO POLITEREFTALATO DE ETILENO RECICLADO EM FIBRAS TÊXTEIS DE POLIÉSTER. .239

\section{ENGENHARIA SANITÁRIA}

O USO DA ENERGIA SOLAR EM HOSPITAIS EM TEMPOS DE PANDEMIA 
ESTUDO DE VARIÁVEIS QUE POSSAM INFLUENCIAR NO CONSUMO DE ÁGUA DA CIDADE DE BAIXO GUANDÚ-ES........

\section{OUTRAS}

SISTEMAAUTOMÁTICO DE IRRIGAÇÃO. .260

A FRUTA DO MILAGRE - SYNSEPALUM DULCIFICUM.. 266 FOSSAS DE EVAPOTRANSPIRAÇÃO: UMA SOLUÇÃO SUSTENTÁVEL PARA OS DEFICITS NO SANEAMENTO EM ÁREAS CARENTES. .271

PROJETO DE ROBÓTICA SOCIAL E EDUCACIONAL .276 


\title{
ESTUDO COMPARATIVO DE FUNDAÇÕES PARA UM EDIFÍCIO MODELO EM JOÃO PESSOA/PB: SAPATA X ESTACA HÉLICE CONTÍNUA
}

\author{
Amanda Sílvia Sousa Ribeiro'; Fernanda Calado Mendonça ${ }^{2}$; Lino Manuel Serra Maia ${ }^{3}$ \\ Engenheira Civil, Faculdade Internacional da Paraíba (FPB), João Pessoa, Paraíba. \\ Professora Mestra em Engenharia Civil, Faculdade Internacional da Paraíba (FPB), João \\ Pessoa, Paraíba; Centro Universitário UNIFACISA, Campina Grande, Paraíba. \\ 14 Doutor Engenheiro Civil, CONSTRUCT-LABEST, Faculdade de Engenharia da \\ Universidade do Porto (FEUP), Portugal; Faculdade de Ciências Exatas e Engenharia, \\ Universidade da Madeira, Portugal.
}

DOI: 10.47094/IJONNE.2021.9

\section{RESUMO}

O objetivo deste estudo é verificar qual tipo de fundação é mais viável para um solo na cidade de João Pessoa. Para isso, foram adotadas cargas pré-dimensionadas do projeto genérico de um edifício comercial e a caracterização do solo foi obtida através de um ensaio SPT. Em posse desses dados, foi possível o dimensionamento de uma sapata retangular e de uma estaca hélice contínua. Foram encontradas as resistências admissíveis para o sistema fundação-solo, para um edifício com quatro e doze pavimentos. Assim, para a proposta do edifício de quatro pavimentos, a sapata se mostrou eficiente em transmitir as cargas para o solo. Já no edifício de doze pavimentos, recorreu-se a um bloco com três estacas do tipo hélice contínua para transmitir de forma satisfatória as cargas solicitantes às camadas resistentes do solo.

PALAVRAS-CHAVE: Fundações. Sapata. Estaca hélice contínua.

ÁREA TEMÁTICA: Engenharia Civil

\section{INTRODUÇÃO}

A escolha da fundação a ser utilizada em um edifício é um dos passos mais importantes do projeto estrutural, pois é esse elemento que tem por finalidade absorver as cargas solicitantes da superestrutura e distribuí-las ao solo. Ao serem executadas apropriadamente, contribuem tanto para uma maior viabilidade econômica da construção quanto a maior durabilidade e segurança, garantindo a estabilidade da estrutura sem recalques excessivos.

A fundação de uma edificação não é o item mais oneroso de uma obra podendo o seu custo 
variar entre 3\% e 7\% do custo total do empreendimento. Apesar disso, erros conceituais de projeto e vícios executivos podem acarretar custos diretos e indiretos elevadíssimos, desde reforços e recuperação estrutural até ações jurídicas de consequências imensuráveis (JOPPERT JR., 2007 apud BEILFUSS, 2012, p. 18).

As fundações podem ser divididas em dois grupos: superficiais (rasas ou diretas) e profundas. Fundações do tipo rasa ou superficial são elementos que transmitem a carga recebida ao solo distribuindo as pressões em sua base, enquanto as fundações do tipo profunda transferem a carga por meio da resistência de ponta (base), pela resistência do fuste (lateral) ou por ambas. A NBR 6122/2019 traz como principais exemplos de fundações superficiais: bloco, sapata e radier; e de fundações profundas: estaca e tubulão.

Por consequência da urbanização, "nos últimos dez anos, a cidade de João Pessoa vem passando por um crescente processo de valorização do solo e de verticalização, tal processo caminha em paralelo com o avanço nos estudos de fundações e análises geológico-geotécnicas" (DUARTE, 2016, p. 12). A modernidade e a evolução econômica e social são grandes responsáveis pelo fenômeno da verticalização, que traz ainda mais à tona a relevância da adoção da fundação adequada.

A solução estrutural apropriada depende do estudo do solo onde a obra será executada. Essa análise auxilia o engenheiro na caracterização do terreno e na estimativa do seu comportamento diante dos esforços solicitantes. Segundo Monteiro (2009 apud BATISTA et al., 2018, p. 15), o ensaio SPT (Standard Penetration Test) permite "a determinação da resistência do solo à penetração, através da amostragem do material ao qual será feito a análise e a determinação das camadas existentes no local onde foi executado a sondagem”, bem como determinar a posição do nível de água.

O presente estudo tem como objetivo verificar qual o tipo de fundação é mais viável para um solo na cidade de João Pessoa; analisar os fatores que influenciam nessa escolha e discutir sobre a viabilidade técnica da fundação escolhida

\section{METODOLOGIA}

Esta pesquisa quantitativa classifica-se como um estudo de caso, pois utilizou um ensaio SPT de um solo da cidade de João Pessoa e um dimensionamento estrutural de uma edificação multifuncional para estimativa de cargas solicitantes necessárias a definição das fundações.

Na primeira etapa, caracterizou-se o solo a partir de um ensaio SPT oriundo do banco de dados de uma empresa especializada no bairro do Aeroclube - João Pessoa com 40,90m de solo sondado. Definindo a carga solicitante referente a apenas um pilar de cada edificação hipotética que será utilizado como referência para a escolha da fundação. Em segundo lugar, seguiu-se o 
dimensionamento, segundo os métodos escolhidos, dos dois tipos de fundações abordados neste estudo: sapata e estaca hélice contínua. Por fim, em um terceiro momento, foi realizada a análise da viabilidade técnica dos resultados encontrados, apontando qual a solução mais adequada para o presente caso.

Para definição de cargas, foi adotado o projeto genérico do edifício comercial encontrado no trabalho de Pires (2011), sendo extraído apenas a carga solicitante por andar do pilar P5 presente na tabela 02 e aplicando essa carga para um edifício de quatro e outro de doze pavimentos.

Em relação ao dimensionamento das fundações, foi utilizado como referência a NBR 6122/2019 - Projeto e Execução de Fundações e bibliografias acerca do tema. Os métodos adotados para determinar a capacidade de carga da sapata e da estaca hélice contínua foram, respectivamente, a Proposição de Vesic à Equação de Terzaghi e o Método de Décourt-Quaresma.

\section{RESULTADOS E DISCUSSÕES}

Através do ensaio SPT utilizado neste estudo, pode-se verificar que as camadas superficiais do solo são caracterizadas por areias siltosas com baixos índices Nspt e, portanto, baixa resistência. Adotou-se o furo 01 para efeitos de cálculo, onde se verifica também um nível alto do lençol freático $(0,83 \mathrm{~m})$. Este perfil é esperado para o solo característico da orla de João Pessoa, região que conta com maior taxa de verticalização na cidade. A orla possui solo arenoso que mescla elementos de sedimentos marinhos e fluviais, no qual o lençol freático tem baixa profundidade (DUARTE, 2016). Sendo assim, a adoção de uma fundação superficial é desaconselhada e pode ser considerada inviável devido ao grau de deformabilidade do solo. As capacidades de carga das fundações foram dimensionadas com o objetivo de atender as cargas solicitantes do pilar P5 dimensionado para um edifício de quatro pavimentos, $\mathrm{Nd}=1040,54 \mathrm{kN}$, e utilizando-se da carga por andar tabelada pelo autor, para um edifício de doze pavimentos, $\mathrm{Nd}=3121,56 \mathrm{kN}$ (PIRES, 2011).

A determinação da capacidade de carga da fundação superficial foi realizada apenas para efeitos demonstrativos, pois constatou-se ao analisar o ensaio SPT do solo que essa solução seria inviável. Foi criada uma hipótese onde o lençol freático estaria na cota de $-3,00 \mathrm{~m}$, possibilitando uma cota de assentamento da sapata retangular rígida em - ,50m. As dimensões adotadas para a sapata foram $B=5,50 \mathrm{~m}$ e $L=4,00 \mathrm{~m}$, gerando um bulbo de tensões $\mathrm{z}=3 \mathrm{~B}$, ou seja, $\mathrm{z}=16,50 \mathrm{~m}$. Essas dimensões foram escolhidas de modo que o bulbo de tensões conseguisse alcançar as camadas mais profundas, com maior índice NSPT e admitindo-se que não haja interferência entre um elemento e outro.

Na Tabela 1 estão reunidos os dados referentes as características da sapata, o bulbo de tensões e a média das camadas de solo dentro do bulbo de tensões (NSPT). Ressalta-se ainda que, por se tratar de um solo arenoso, não possui coesão $(c=0)$, com peso específico $(\gamma)$ de $18 \mathrm{kN} / \mathrm{m}^{3} \mathrm{e}$ sobrecarga q $=\gamma$ h (ALBIERO; AOKI; CINTRA, 2011, p. 26). 
Utilizando-se da Proposição de Vesic, extraiu-se os valores dos fatores de capacidade do solo e fatores de forma. Além disso, foi empregado o fator de segurança igual a 2,0 como indicado pela NBR 6122/2019. De forma que foram obtidos os valores da capacidade de carga calculada e a capacidade de carga admissível iguais a $2828,19 \mathrm{kN}$ e $1414,10 \mathrm{kN}$ respectivamente.

Os valores da capacidade de carga para fundação do tipo hélice contínua foram encontrados através do Método de Décourt-Quaresma, compostos pela resistência lateral e pela resistência de ponta. $\mathrm{Na}$ Tabela 2, foram reunidas as características adotadas para a estaca hélice contínua, além da altura do bloco de coroamento, o diâmetro da estaca, a área da seção transversal da ponta, o perímetro da seção transversal do fuste e os fatores de adesão entre solo e estaca $\alpha$ e $\beta$.

Tabela 1: Dados referentes à sapata e ao solo.

\begin{tabular}{|l|l|r|}
\hline SAPATA RETANGULAR RÍGIDA & \multicolumn{2}{|l|}{} \\
\hline DIMENSÕES $(\mathrm{m})$ & B & 5,50 \\
\hline & L & 4,00 \\
\hline BULBO DE TENSÕES $(\mathrm{m})$ & & 16,50 \\
\hline MÉDIA NSPT & & 17,00 \\
\hline ÂNGULO DE ATRITO $(\phi)$ & & 34,80 \\
\hline PESO ESPECÍFICO $\left(\mathrm{kN} / \mathrm{m}^{3}\right)$ & & 18,00 \\
\hline $\mathrm{q}(\mathrm{kPa})$ & & 27,00 \\
\hline
\end{tabular}

Fonte: Autoria própria (2020)
Tabela 2: Dimensões e características da estaca hélice contínua.

\begin{tabular}{|l|r|}
\hline \multicolumn{2}{|l|}{ ESTACA HÉLICE CONTÍNUA } \\
\hline ALTURA DO BLOCO $(\mathrm{m})$ & 1,00 \\
\hline DIÂMETRO & 0,50 \\
\hline ÁREA DA SEÇÃO CIRCULAR $\left(\mathrm{m}^{2}\right)$ & 0,20 \\
\hline PERÍMETRO $(\mathrm{m})$ & 1,57 \\
\hline$\beta$ & 1,00 \\
\hline$\alpha$ & 0,30 \\
\hline
\end{tabular}

Fonte: Autoria própria (2020)

Em posse desses dados, foram calculadas as resistências: lateral (RL), de ponta (RP), total (RT) e a total admissível (Radm), sendo esta encontrada através da utilização do fator de segurança $\mathrm{FS}=2$. Os valores da resistência total admissível ultrapassaram o valor de $1000 \mathrm{kN}$ a partir dos 16 $\mathrm{m}$ de profundidade, horizonte que foi considerado neste trabalho para análise. A partir do ensaio SPT, é possível constatar um solo com maior deformabilidade, que deve sustentar dois pilares prédimensionados com cargas solicitantes de cálculo $\mathrm{Nd}=1040,54 \mathrm{kN}$ (prédio de quatro pavimentos) e $\mathrm{Nd}=3121,56 \mathrm{kN}$ (prédio de doze pavimentos).

Ao fazer um levantamento sobre as fundações mais utilizadas nos solos litorâneos da cidade de João Pessoa, Duarte (2016) ressalta que uma solução comum adotada seria o reforço da fundação com estacas de compactação, aumentando a capacidade de carga do solo. Esse método é bastante utilizado em obras de pequeno porte e de localização mais próxima da orla, devido a maior viabilidade econômica.

Sendo assim, o sistema sapata-solo é viável apenas para as cargas do pilar referente ao edifício de quatro pavimentos. Vale ressaltar que essa capacidade de carga foi calculada para um nível de lençol freático hipotético, pois a existência de água no solo inviabilizaria a execução da sapata, além da presença de areia fofa nas cotas de assentamento (SILVA, 1998). Nestes casos, existem alternativas como a adoção de técnicas de rebaixamento do nível do lençol freático ou, como dito anteriormente, o reforço da fundação com estacas de compactação. 
Em se tratando de obras maiores e, consequentemente, na maior solicitação de cargas, as fundações diretas deixam de ser uma opção viável. Com os resultados obtidos foi possível verificar que uma solução de três estacas hélices contínuas por bloco de coroamento consegue, de forma satisfatória, resistir ao pilar de maior carga solicitante e que a camada resistente não se encontra muito distante da superfície.

Para além das cargas solicitantes, o nível do lençol freático na cota $-0,83$ m reforça a necessidade da adoção de uma fundação profunda. A estaca hélice contínua se mostra vantajosa, pois pode ser executada em solos com alto nível de lençol freático e possui uma execução com menos ruído e vibração. Isso possibilita a utilização desta em áreas residenciais e causa pouca ou nenhuma perturbação em obras vizinhas (NETO, 2002).

\section{CONSIDERAÇÕES FINAIS}

Conforme exposto, a fundação é um elemento de vital importância para a segurança e estabilidade da obra. São vários fatores que devem ser levados em consideração no momento da escolha de um sistema fundação-solo que tenha a capacidade de resistir de forma mais eficiente à superestrutura. Dentre esses, ressalta-se o estudo do solo através do ensaio SPT, o conhecimento das obras vizinhas, as cargas solicitantes dos pilares e os aspectos técnicos dos tipos de fundações.

Para a proposta do edifício de quatro pavimentos, a fundação superficial em sapata se mostrou eficiente em transmitir as cargas para o solo. Sua limitação ocorreu em face do nível do lençol freático, que compromete a execução desse tipo de fundação no solo estudado. Como solução, recorreu-se a um levantamento das fundações mais executadas em solos similares, encontrando como mais comum o reforço da fundação com estacas de compactação.

Já no edifício de doze pavimentos, o sistema sapata-solo não foi capaz de atender as cargas solicitantes. Para este, a solução adotada foi a fundação profunda em estaca de hélice contínua, pois ela não possui restrições quanto ao nível do lençol freático em sua execução, assim como pode ser utilizada em áreas residenciais por não apresentar altos níveis de ruídos ou perturbações as obras vizinhas.

Tais resultados se mostraram coerentes e apropriados para o perfil de solo estudado, onde pode-se verificar que as fundações superficiais podem ser soluções tecnicamente viáveis para obras de pequeno porte mesmo em solos menos resistentes, comumente encontrados na orla da cidade. 


\section{FINANCIAMENTO}

Financiamento Base - UIDB/04708/2020 ao CONSTRUCT - Instituto de I\&D em Estruturas e Construções - financiado pelos fundos portugueses através de FCT/MCTES (PIDDAC). Financiamento Português através da FCT - Fundação para a Ciência e a Tecnologia, I.P., dentro do Estímulo do Emprego Científico - Concurso Institutional - CEECINST/00049/2018.

\section{REFERÊNCIAS}

ALBIERO, José Henrique; AOKI, Nelson; CINTRA, José Carlos A. Fundações diretas: Projeto geotécnico. São Paulo: Oficina de Textos, 2011.

AOKI, Nelson; CINTRA, José Carlos A. Fundações por estacas: Projeto geotécnico. São Paulo: Oficina de Textos, 2010.

BEILFUSS, Tatiane. Estudo comparativo da fundação de um edifício modelo: Estaca $\mathbf{x}$ tubulão. 2012. Trabalho de Conclusão de Curso de Engenharia Civil - Universidade Regional do Noroeste do Estado do Rio Grande do Sul, Ijuí, 2012.

DUARTE, Marianna Mesquita. Prática das fundações na cidade de João Pessoa. 2016. Trabalho de Conclusão de Currso de Engenharia Civil - Universidade Federal da Paraíba, João Pessoa, 2016.

NETO, José Albuquerque de Almeida. Análise do desempenho de estacas hélice contínua e ômega: Aspectos executivos. 2002. Dissertação (Mestrado em Engenharia) - Escola Politécnica, Universidade de São Paulo, São Paulo, 2002

PIRES, Gustavo Menegusso. Estudo do pré-dimensionamento de pilares. 2011. Trabalho de Conclusão de Curso de Engenharia Civil - Universidade Regional do Noroeste do Estado do Rio Grande do Sul, Ijuí, 2011.

SILVA, Edja Laurindo da. Análise dos modelos estruturais para determinação dos esforços resistentes em sapatas isoladas. 1998. Dissertação (Mestrado em Engenharia de Estruturas) - Escola de Engenharia de São Carlos, Universidade de São Paulo, São Carlos, 1998. 


\section{editoraomnisscientia@gmail.com M} https://editoraomnisscientia.com.br/

@editora_omnis_scientia @ https://www.facebook.com/omnis.scientia.9 f

$$
\text { +55 (87) 9656-3565 @ }
$$




\section{editoraomnisscientia@gmail.com M} https://editoraomnisscientia.com.br/ $\oplus$

@editora_omnis_scientia@ https://www.facebook.com/omnis.scientia.9 f

$$
\text { +55 (87) 9656-3565 @ }
$$

